\title{
Sulla tossicità di alcuni propellenti per missili $\left(^{*}\right)$
}

\author{
G. LALLI (**)
}

Ricevuto il 19 Febbraio 1963

\begin{abstract}
Riassunto. ... In missilistica trovano impiego propellenti ed ossidanti dotati di notevole tossicità. Tra i primi si ricorda l'ammoniaca, l'anilina, gli alcooli (etilico, metilico, forfurilico), lo $\mathrm{J}_{\mathrm{p}} 4$, i borani, i derivati metilati dell'idrazina. Tra i secondi il perossidlo di idrogeno, l'ossigeno liquido, l'acidlo nitroso nitrico fumante ece.

I principali aspetti tossicologici ed igienico-sanitari derivati dall'impiego di molte di tali sostanze, alcune delle quali trovano estese applicazioni nelindustria, sono ornai sullicientemente chiariti dallampia letteratura in argomento. Par tale motivo l'A. circoscrive la propria esposizione ai borani ed ai derivati metilati dell'idrazina, dal cui impiego, praticamente confinato al settore missilistico, possono derivare all'organismo dami meno largamente conoscinti, e, sotto certi aspetti, tuttora abbisognevoli di ulteriori chiarimenti.

Per tali sostanze vengono riferite le principali caratteristiche fisiche e chimiche, le vie di penetrazione nell'organismo, $i$ dati relativi alla loro tos. sicita acuta, sub-acuta e cronica, con particolare riguardo alle concerntrazioni al limite di tossicità, la sintomatologia dell'intossicarione e, per concludere, le principali norme preventive.
\end{abstract}

SUmury. - Some of the propellauts and oxidizer used for missiles present marked toxic properties. In the first groul we may cite ammonia, aniline, alcohols (ethyl, methyl and furfurilic alcohols), $\mathrm{J}_{1} 4$, boranes, and lyydrazine methyl derivatives; in the second group hydrogen peroxide, liquid oxygen, fuming nitrous-nitric acirl, etc.

The major issues in respect of the toxicological and hygrienic aspects rolating to the use of these substances, some of which are also extensively

(*) Nota presentata al $2^{\circ}$ Congresso Internazionale Teenico Scientifico dello Spazio. Roma, 19-23 Giugno 1962.

(**) Ispettorato di Sanita Aeronautica. Capo dell' Ispettorato: T'en. Gen. Medico C.s.A. Prof. Dott. T. Lomoxico.

Centro di Studi e Ricerche di Medicina Aeronautica espaziale. Direttore: 'T'n. Col. Medico (‥A. Prof. Dott. A. Scano 
used in industry, have already been exhanstively dealt with in the relevant literature. For this reason the author has confined his study to boranes and to the methylhydrazine derivatives: the damages which these products, which are practically used only in connection with missiles, can produce in the luman body are less well known and in some respects need further clarification.

'The author indicates their major physical and chemical properties, the channels through which they penetrate the human body and the data pertaining to the acute, sub-acute and chronic poisoning, with particular reference to concentrations standing at the limits of toxicity, intoxication symptoms and the most important methods of protection from their larmful effects.

RÉsunÉ - Dansl e domaine de la navigation siderale l'on fait recours à l'emploi de propergols et d'oxydateurs ayant un degré toxique assez élevé. Parmi les premiers nous rappelons l'ammoniaque, l'aniline, certains alcohols (éthylique, méthylique, furfurique). le Jp 4, les boranes, les dérivés méthylés de l'hydrazine. Parmi les autres, le péroxyde d'hydrogène, l'oxygène liquide, l'acide nitrique, etc.

Les aspects toxicologiques principaux ainsi que les aspects sanitaires et d'hygiène découlant de l'emploi de beaucoup de ces matières dont quelques-unes sont très employés dans l'industrie, sont désormais suffisamment éclaircis grâce ì la littérature exhaustive en la matière. ("est pourquoi l'Auteur limite son exposé à des boranes et à des dérivés méthylés de l’hydrazine, dont l'emploi limité pratiquement an domaine des missiles, peut déclencher dans l'organisme des dommages moins connus appelant, du moins sous certains aspects, davantage de clarté.

L Auteur expose les caractéristiques principales physiques et chimiques de ces matières, leurs voies d'accès à l'organisme les domnées concernant leur caractère toxique aigu, sub-aigu et chronique, eu égard spécialement aux concentrations qui ont à la limite de ce caractère toxique. Il étudie ensuite la symptomatologie de l'intoxication et fualement les principales normes sanitaires de prévention.

\section{Premessi.}

I missili comunque guidati esigono carburanti capaci di sviluppare in brevissini periorli di tempo enormi quantità di energia in piceole amere di combustione. Sono quindi necessari combustibili dotati di notevole energia e capaci di reagire pressoché istantaneamente con sostanze ossidanti. Alcuni di tali carburanti ed ossidanti ed i loro prodotti di decomposizione sono estremamente tossici per l'uomo con conseguenti piǹ o meno gravi rischi per il personale addetto alla loro produzione, 
al loro immagazzinamento, al loro impiego. Ne derivano problemi profilattici di notevole interesse pratico per la salvaguardia di detto personale.

Nel settore dei propellenti il problema tossicologico acquista particolare rilievo per l'ammoniaca, l'anilina, gli alcooli (etilico, metilico, furfurilico) per lo JP-4, per i borani, per i derivati metilati dell'idrazina; nel settore degli ossidanti per il perossido di idrogeno, per l'ossigeno liquido e per l'acido nitroso-nitrico fumante. Per molte di tali sostanze, alcune delle quali trovano estese applicazioni nell'industria, sussiste ormai un'ampia letteratura, sufficiente a chiarire i principali aspetti tossicologici ed igienico-sanitari derivanti dal loro impiego.

Per tale motivo circoscriviamo la nostra esposizione ad una breve rassegna della letteratura concernente $\mathrm{i}$ borani ed i derivati metilati dell'idrazina sia perchè trattasi di propellenti il cui impiego è prevalentemente confinato alla missilistica, sia perchè dal loro uso possono derivare all'organismo danni meno largamente conosciuti e, sotto certi aspetti, tuttora abbisognevoli di ulteriori ehiarimenti.

\section{BORINI}

Sono composti di boro ed idrogeno (idruri di boro). Quali propollenti ad alto valore energetico sono soprattutto impiegati il diborano, il pentaborano ed il decaborano.

CARITTERISTICHE CHIMICo-Fisiche.

Il diborano $\left(\mathrm{B}_{2} \mathrm{I}_{6}\right)$ in condizioni, ordinarie di temperatura e pressione si presenta come un gas incolore, il cui punto di ebollizione è $-92,5 \circ \mathrm{C}$, dall'odore nauseante di uova marce, apprezzabile alla concentrazione di $3,3-4,2 \mathrm{mg} / \mathrm{mc}$.

In contatto con l'acqua si idrolizza in pochi secondi con produzione di acido borico (borati), idrogeno e con sviluppo di una notevole quantità di calore.

Poiché l'acido borico e l'idrogeno sono relativamente poco tossici per l'nomo è probabile che l'azione tossica della sostanza sia almeno in parte collegata alla predetta reazione esotermica che si produce nelle vie respiratorie.

Il pentaborano $\left(\mathrm{B}_{5} \mathrm{H}_{0}\right)$ è un liquido molto volatile, con punto di ebollizione a $+58 \circ \mathrm{C}$, dall'odore difficilmente definibile, leggerments 
dolciastro, agliaceo, simile all'acetilene, approzzabile alla concentrazione $1,7-3,6 \mathrm{mg} / \mathrm{mc}$.

La completa idrolisi in acqua richiede molte ore alla temperatura del rorpo umano con formazione degli stessi prodotti cui dà origine il diborano.

Il decaborano $\left(\mathrm{B}_{10} \mathrm{H}_{14}\right)$ è un solido cristallino volatile il cui punto di fusione d 99,7 oC, di colore bianco, di odore spiacevole, pieno, che ricorda il ciocolato, apprezzabile a concentrazioni estremamente basse $(0,24$ $0,49 \mathrm{mg} /(\cdot \mathrm{ml})$.

Sebbene sia insolubile in acqua, l'idrolisi completa della sostanza si attua in rilca 30 giomi.

\section{DOSAGGIO DHI BORANI NEI MLATERIALI BIOLOGICI.}

Sumerosi sono i procedimenti proposti per il dosaggio del boro nei materiali biologici (Ellis a Coll., 1949; Hatcher e Wilcox, 1950; Dible e Coll., 1954; Naftel, 1939; Hill a Coll., 1958). Tutti hamno l'inconveniente di una magrgione o minore complessità nonché quella di esigere laboratori particolarmente attrezzati o dotazioni di strumenti specializzati: inoltre essi dosano tutto il boro presente senzal poter differenziare i valori del boro di fondo di provenienza alimentare (vari vegetali, acqua, etc.) che possono laggeiumgere valori notevolnente alti $(17 \mathrm{mg} / 100 \mathrm{gr}$ nellar coute, da 0,8 a $3,23 \gamma / \mathrm{ml}$ nel sangue secondo Levinskase e Coll., 1956) e soprattutto variabili, da quello contenuto nei borani.

Una semplice tecnica per il dosaggio di questi ultimi è quella proposta da Miller e Coll., 1959, eapace di svelare sino a 0,05 gamma di boro per ml di siero con una riproducibilita del $\pm 5 \%$ e che consiste nella preliminare estrazione del siero con alcool-etere, che elimina it boro di fondo di provenienza dietetica, e nel successivo dosagerio colorimetrico del solo boro contenuto nei borani (che, essendo legato alle lipoproteine, rimane in soluzione) mediante la reazione alla curcumina.

Sin dal 1870 è noto che la tintura di curcumina quando viene evaporata in presenza di acido borico forma un complesso colorato. L'agente attivo, ot tenuto anche per sintesi, è $1, \tau$ bis (4-idrossi-3 metossifenil)-1,6 eptadiene-3,5 dione. Il complesso boro-curcumina esiste in 2 forme (Clarke e Jackson, 1908; Apicer a Strickland, 1952): la rosocianica che consiste in due molecole di curcumina riunite da un solo atomo di boro e la rubrocuremmina nela quale il rapporto boro/curemina è pari ad 1. Tali complessi hanno caratteristiche similari presentando il loro massimo 
assorbimento a 5.50 $111 \mu$ e si formano, nelle conclizioni volute dal metolo, pressoché in parti egguali.

Oltreché con boro, la rulcumina forma complessi colorati ron il berillio, il magnesio, il molibeleno, il titanio, lo zireonio, l'uranio, il bario, l'hatuio, il tungsteno, il gemanio a lo ione ferrico.

Di tali celementi solo lo ione ferrico è presente nei mammiferi in quantita discrete: fortunatamente la sua concentrazione nel siero non it sufliciente ad interferine.

Il closaggio del solo boro con il test alla eureumina puó essere facilmente conseguito mediante aggiunta di aleali che riducono gli altri complessi allo stato originario: tuttavia tale riduzione non è necessaria in condizioni sperimentali ordinarie nei materiali biologiei animali.

Sostanze fortemente ossidanti, come perossidi, cromati, permanganati e clorati possono interferire nello sviluppo del colore. Pertanto nellestrazione ì necessialio impiegare solo etere privo di perossidi e vetreria scrupolosamente pulita.

\section{Reuttivi.}

1) Miscelat alcool-etere: si prepara mescolando a parti eguali alcool assoluto ed etere dietilico privo di perossidi.

2) Reattivo alla cureumina: in pallono tamato da $100 \mathrm{sc}$ sogliere $10 \mathrm{gr}$ di acido ossalico purissimo per analisi in circar $70 \mathrm{ml}$ di alcool etilico assoluto. Aggimgere quindi gr 0,1 di courcumind ricristallizzata (è fornita da Hatheson Coleman e Bell, East Rutherford, New Jersey); $10 \mathrm{ml}$ di ancido cloridrice concentrato purissino. Portare a volume con alcool assoluto. Lal stabilità è di almeno 1 mese a temperatura ambiente.

\section{Tecuica.}

1) Spipettare $1 \mathrm{ml}$ di siero non emolizzato in 5 $\mathrm{ml}$ di miscela alcooletere in un tubo da centrifuga di $15 \mathrm{ml}$.

2) Allestire parimenti un controllo el un bianco con 1 ml di siero di soggetto non esposto ed $1 \mathrm{ml}$ di $\mathrm{H}_{0} \mathrm{O}$ distillata.

3) Porre i lubi in bagno maria e far bollire per 30". Lar sopracbollizione va evitata agitando costantemente i tubi con l'ainto di una bacrhettina di votro.

4) Centrifugare per $3^{\prime}$ a 1000 giri.

5) Senzar disturbare il precipitato proteico aggiungere 0,5 ml di reattivo alla curcumina in ciascun tubo. 
6) Decantare i soplanatanti in altrettanti ansule di vetro o porcellana ed agitare.

7) Essiccare in stufa a secco sino a completo essiccamento. La tempelatula dovrà essere molto vicina a 53 oC senza però mai superarlal.

8) Togliere le capsule dalla stufa ed estrarre il complesso colomato con aliquote di $5 \mathrm{ml}$ di alcool etilico a $95^{\circ}$ con l'aiuto di ma bacchetta di vetro riunendo le diverse aliquote sino ad un volume finale di $20 \mathrm{ml}$.

9) Leggere la densità ottica a $540 \mathrm{~m} \mu$ azzerando con la prova in bianco. Il siero di controllo negativo non dovrà fornire valori superiori a $0,05 \gamma / m 1$.

\section{Taratura.}

Può essere effettuata con soluzione di acido borico. Allestire all'nomo la soluzione contenente $0,1 \mathrm{mg} / \mathrm{ml}$ di boro sciogliendo $0,5714 \mathrm{mg}$ di acido borico purissimo in $1000 \mathrm{ml}$ di acqua distillata. Da questa allestire per diluizione soluzioni contenenti da 0,01 a $3 \gamma / \mathrm{ml}$ di boro. Trattare dette soluzioni come prima indicato per il siero. La curva di taratma, sino ald oltre $3 \gamma / \mathrm{ml}$ di boro, è una leetta.

Il procedimento descritto (Miller e Coll., 1960) può essere, con qualche variante, adatiato al dosaggio dei borani nei tessuti (fegato, cervello).

Per il fegato si consiglia di estralre e successivamente di tratiare con il reattivo alla ceureumina $2 \mathrm{ml}$ di un omogenato ottenuto da parti uguali di tessuto epatico ed acqua distillata.

Per' il cervello $1 \mathrm{gr}$ di tessuto viene bollito con $10 \mathrm{ml}$ di reattivo alla curcumina. Dopo essiceamento si procede come indicato per il siero.

\section{'TOSSICOLOGIA SPERIMEN'TALE.}

La farmacologia dei borani derivata soplattutto dagli studi animali la conclotto a risultati che evidentemente forniscono ma guida ma che non possono essere indiscriminatamente extrapolati all'nomo.

Gli studi sperimentali eflettuati hanno commoue condotto alla dimostrazione che i tre idruri di boro soprec considerati e cosi pure i propellenti ad alta energia da essi derivati sono tutti dotati di elevatissima tossicità. Di essi il decaborano è il meno tossico; segue il diborano che è, secondo Kunkel, 1950, da 15 a 150 volte piǹ tossic'o dell'acido borico e la cui tossicità è dell'ordine di glandezza di quella del fosgene. La tossicità del pentaborano, che è ancora più pericoloso, può essere comparata ed è anzi superiore a quella dell'acido cianidrico. 
Tra il diborano da un lato ed il penta e decaborano dall'altra esistono nette differenze nel meccanismo di azione, e quindi nella sintomatologia.

L'intossicazione acuta da diborano è infatti caratterizzata da fatti irritativi a carico delle prime vie aeree che conducono, nei casi più gravi, all'edema polmonare ed alla morte (Krackow, 1953, Kunkel e Coll. 1956). Analoghi sintomi irritativi con diminuita resistenza alle infezioni respiratorie caratterizzano l'intossicazione sub-acuta o cronica: tali fatti sono presumibilmente in rapporto alla pressoché istantanea reazione esotermica idrolitica che il prodotto subisce al suo contatto con le mucose.

Il deca ed il pentaborano, anche se introdotti per via inalatoria non producono lesioni respiratorie, ma agiscono soprattutto sul S.N.C. o, subordinatamente su altri organi (fegato, rene, ecc.) producendo quindi sugli animali da esperimento astenia, incoordinazione motoria, convulsione, coma, morte. Poco noto è tuttora il meccanismo genetico di tali intossicazioni: ̀̀ tuttavia da tenersi presente che la reattività chimica dei borani puó essere attribuita alla proprietà del boro atomico di fissare elettroni: ne deriva che i borani sono dotati di proprietà fortemente riducenti (Stock, 1933; Hurd, 1952); reagendo facimente con ammoniaca, amine organiche, idrocarburi insaturi, varie amine eterocicliche, ece. il che giustifica molteplici possibilità di interferenze nei processi biochimici. E inoltre da ricordare che dal diborano può prodursi pentaborano e che da quest'ultimo possono originarsi vari idruri di boro, tra cui il decaborano.

La penetrazione dei borani nell'organismo può attuarsi per via inalatoria, per via percutanea e per ingestione o parimenti altamente tossici essi si dimostrano se sperimentalmente inoculati per via parenterale. La gravità degli effetti che derivano da singole esposizioni acute o da ripetute esposizioni sub-acute è soprattutto legata ai dosaggi impiegati, mentre importanza relativamente modesta compete alla vita ed alla modalità della somministrazione; ciò è valido soprattutto per la via inalatoria e per quella parenterale, mentre per quella percutanea i dosaggi necessari a conseguire $i$ medesimi effetti sono più elevati.

\section{Tossicità acuta.}

La tossicità acuta di tali sostanze è sufficientemente chiarita dati numerosi studi in argomento (Wills, 1953; Krackow, 1953; Svibely, 1954, $195 \check{5}, 19506$, есc.).

La $\mathrm{LI}_{50}\left(\mathrm{~V}\right.$. Tabelle $\mathrm{n}^{\mathrm{o}} 1$ e 2 ) per via inalatoria e per esposizione di 4 ore è pari a circa 50 ppm per il ratto (Krackow); per esposizione di 
2 ore, con morte degli animali nello stesso periodo di tempo, è di 19 ppm per il pentaborano, di $25,7 \mathrm{ppm}$ per il decaborano. $\mathrm{L}_{a} \mathrm{~L}_{\mathbf{5} 0}$ riferita per quest'ultima sostanza, con morte degli animali (latti) entro 48 ore è di $32 \mathrm{mg} / \mathrm{kg}$ per via entroperitoneale; di $40 \mathrm{mg} / \mathrm{kg}$ per via orale; di $71 \mathrm{mg} / \mathrm{g} \mathrm{kper} \mathrm{applicazioni} \mathrm{cutanee.}$

Tabella 1 - Tossiciti aCUTA DEL PENTABoraxo E DEL DECABoraxo intio-

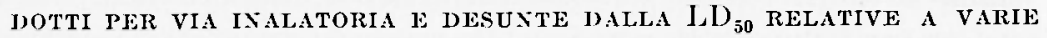
CONDIZIONI SPERIMENTALI IN TOPI E RATTI DI SESSO MASCIILE (RICAVATA DAI DATI DI SVIRBELY E, PER IL DiboraNo, Da KRACKow).

\begin{tabular}{|c|c|c|c|c|c|c|c|}
\hline \multirow{3}{*}{ Sostanza } & \multirow{3}{*}{\multicolumn{3}{|c|}{ Condizioni sperimentali }} & \multicolumn{4}{|c|}{$L, I_{50}$} \\
\hline & & & & \multicolumn{2}{|c|}{ 'Topo } & \multicolumn{2}{|c|}{ Ratto } \\
\hline & & & & $\mathrm{ppm}$ & $\mathbf{m} \mathrm{g} / \mathrm{ml}$ & ppm & $\mathrm{mg} / \mathrm{ml} 1$ \\
\hline Pentaborano & Esposiz. per $2 \mathrm{l}$ & I. Mor & ntro $2 \mathrm{~h}$ & 14,1 & 0,037 & 19,5 & 0,051 \\
\hline Decaborano & "2, & 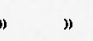 & $2 "$ & 35,6 & 0,182 & 25,7 & 0,122 \\
\hline Pentaborano & $" 2$ & $"$ & $" 4 n$ & 10,9 & 0,028 & 17,8 & 0,046 \\
\hline Decaborano & ”2, & $"$ & $4 "$ & 35,6 & 0,182 & 25,7 & 0,122 \\
\hline Diborano & $" \quad 4$ & & & - & - & 50 & - \\
\hline
\end{tabular}

La sintomatologia dell'intossicazione acuta degli animali (cane, coniglio, ratto, topo) è eminentemente variabile in rapporto soprattutto alla concentrazione del tossico ed alla durata dell'esposizione.

In cani esposti per periodi di tempo variabili da 1 a 4 ore a piccole concentrazioni di diborano (da 7 a $15 \mathrm{mg} / \mathrm{mc}$ ) per via inalatoria si ha

Tabella 2 - Tossicitì acuta deI decaborajo per via intraperitoneaLE, ORALE E PERCUTANeA DESUNTA DALLA LD SEsSo mascille (RICAVATA Dai Dati di SVIRBELY).

\begin{tabular}{|c|c|c|c|c|c|c|c|}
\hline \multirow{3}{*}{$\begin{array}{l}\text { Via di in- } \\
\text { troduzione }\end{array}$} & \multirow{3}{*}{$\begin{array}{l}\text { Dopo } \\
\text { osser. } \\
\text { di ore }\end{array}$} & \multicolumn{6}{|c|}{$\mathrm{LD}_{50} \mathrm{mg} / \mathrm{kg}$} \\
\hline & & \multicolumn{2}{|c|}{ Topo } & \multicolumn{2}{|c|}{ Ratito } & \multicolumn{2}{|c|}{ Coniglio } \\
\hline & & media & da... a... & media & da... a... & media & da... a... \\
\hline Intraperit. & 24 & - & 一 & 27 & $24,7-29,5$ & - & - \\
\hline$”$ & 48 & 33,2 & $30,5-36,2$ & 23,4 & $21,4-25,6$ & - & - \\
\hline Orale & 48 & 40,9 & $33,4-50,2$ & 64,3 & $57,5-71,8$ & - & - \\
\hline Appl. cut. & 24 & - & 一 & 890 & $812-976$ & 113 & $51-247$ \\
\hline$"$ & 48 & 一 & 一 & 740 & $676-809$ & 71 & $32-155$ \\
\hline
\end{tabular}


solo un lieve aumento della frequenza respiratoria. Dosaggi più elevati $60-70 \mathrm{mg} / \mathrm{me}$ per $\left.15^{\prime}\right)$ producono in più lieve caduta della pressione arteriosa con diminuzione della frequenza cardiaca.

All'autopsia si nota presenza di congestione e di emorragie ai polmoni, ed, in qualche caso, edema polmonare; congestione a carico dei visceri; lesioni renali caratterizzate dalla presenza di cilindri nei tubuli. Dosaggi ancora più elevati $\left(400 \mathrm{mg} / \mathrm{mc}\right.$ per $\left.15^{\prime}\right)$ producono rapida caduta della pressione arteriosa, notevole aumento della frequenza respiratoria; netta bradicardia con aumento, all'elettrocardiogramma, dell'onda $\mathrm{T}$ : la morte interviene in pochi minuti per edema polmonare, di cui si ha conferma all'esame anatomopatologico.

La sintomatologia dell'intossicazione acuta da penta e decaborano è prevalentemente a carico del S.N.C. Una analisi dettagliata di essa in rapporto ai dosaggi, alla via di somministrazione ed all'animale impiegato può ritrovarsi nei lavori di Svirberly.

I fenomeni più frequentemente osservati (ratto, topo) nell'intossicazione da pentaborano consistono in convulsioni, tremori, protrusione degli occhi, lenti movimenti degli arti anteriori e posteriori, irrequietezza; tendenza degli animali a strisciare cantamente mentre sono in preda ai tremori e ad ammucciarsi, stato stuporoso, coma, morte, talora improvvisa, talora con convulsione ed eiaculazione. Post mortem si può avere distensione addominale e, frequentemente, comparsa di opacità corneali che intervengono qualsiasi sia stata la via di introduzione del tossico per motivi che permangono tuttora ignoti.

Di regola negli animali sopravissuti persistono abbastanza a lungo tremori e stato itterico. La restitutio ad integrum dell'intossicazione sperimentale da pentaborano è più rapida (diverse ore) se comparata con gli effetti protratti del decaborano (diversi giorni).

La sintomatologia negli animali che hanno avuto contatto con quest'ultima sostanza è analoga: i bassi dosaggi producono convulsioni transitorie, disattenzione, dispnea, tolleranza (nel topo e nel ratto) alla posizione sdraiata: rilessatezza, tremori convulsivi, movimenti rotatori rapidi delle zampe. Con i dosaggi più elevati si hanno spasmi transitori, dispnea, rilassatezza, coma, morte. Eे importante il fatto che a tali dosaggi mancano per lo più i fenomeni convulsivi, mentre prevalgono quelli depressivi e la morte è preceduta da stato comatoso. Negli animali sopravvissuti residuano persistenti secrezioni delle congiuntive oculari e dalle narici. Anche qui è frequente la comparsa di opacità corneali post-mortem.

I borani, per qualsiasi via introdotti, passano lentamente nel sangue dove sono reperibili in quantitativi progressivamente crescenti e la gra- 
vita della sintomatologia da essi prodotta è (Miller e Coll., 1960) strettamente correlata al tasso serico del tossico.

Il fenomeno risulta chiaramente dalla Fig. 1 che dimostra la corrclazione esistente nel cane tra la sintomatologia ed il tasso del boro dopo introduzione per via intraperitoneale di una dose letale $(15 \mathrm{mg} / \mathrm{kg}$.) di un propellente boranico (II E F-3). Tassi serici dell'ordine dei $3 \gamma$ si associano a sintomatologia gravissima con imminente pericolo di vita.

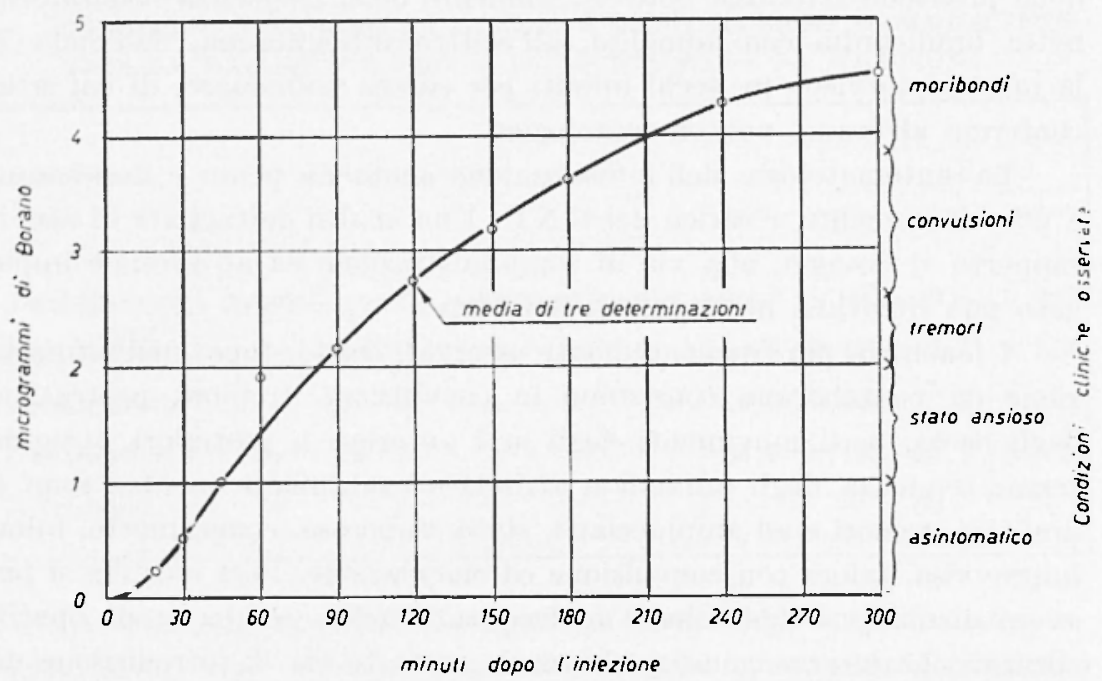

Fig. 1 - Valori del borano serico e corrispondenti condizioni cliniche dopo somministrazione, nel cane, di una dose acutamente letale di HEF - 3. (da Miller, 'Tamas, Robinson, Merriweather, 1960)

I reperti anatomo-patologici nell'intossicazione sperimentale acuta da diborano sono stati prima descritti. In quella da penda e decaborano l'esame autoptico mette in evidenza ingrossamento del fegato, la cui superficie appare ruvida e mammellonata, di colorito giallo bruno, congestione dei visceri, in specie dei polmoni; surreni di colorito bruno; presenza di gas nell'intestino. Istologicamente si ha reperto di lieve glomerulo-nefrite, con congestione e grave rigonfiamento torbido a carico dei tubuli renali; edema polmonare emorragico; disorganizzazione tossica dei nodi linfatici; mareata distruzione del parenchima epatico che non è più riconoscibile.

Mancano al momento esaurienti descrizioni anatomo-patologiche relative al sistema nervoso. 


\section{Tossicità sub-acuta.}

Interessanti contributi alla conoscenza dell'intossicazione sub-acuta da borani sono soprattutto dovuti a Svirbely (1954-1955) ed a Miller e Coll. (1960).

Il primo ha immanzitutto documentato l'azione cumulativa prodotta dalla somministrazione di piccoli dosi giomaliere di recabomo (introdotto per inalazione, per iniezione intraperitoneale, per somministrazione orale e per applicazioni percutanee nel ratto nel coniglio e nel ane) e di pentaborano (introdotto per via inalatoria). Ha inoltre descritto la sintomatologia ed il decorso dell'intossicazione sub-acuta sperimentale, corredata da alcuni dati di laboratorio e dai reperti autoptici.

Da tali studi risulta in definitiva che:

1) successive somministrazioni quotidiane, largamente inferiori a.la $\mathrm{LD}_{50}$ stabilita per unica somministrazione acuta, conclucono all'intossicazione ed alla morte degli animali;

2) la sintomatologia dell'intossicazione sub-acuta è discretamente variabile in rapporto alla sostanza ed ai dosaggi impiegati, alla via di somministrazione ed all'animale in esperimento. Gli effetti sul S.N.C. appaiono di massima accentuati a paragone con quelli che si osservano dopo esposizioni singole. I seguenti sintomi sono frequentemente osservati: disattenzione, nervosismo, atteggiamento aggressivo, convulsioni, grossolani tremori della testa, perdita considerevole di peso, salivazione, vomito anoressia, dilatazione dei vasi congiuntivali. Può inoltre aversi tachipnea e tachicardia e, nei casi più gravi, bradipnea e bradicardia, che precedono il coma e la morte.

Gli effetti sul S.N.C. sono molto marcati nel ratto sottoposto all'intossicazione da pentaborano per via inalatoria; meno aceentuati invece nel coniglio e nel cane dopo somministrazione di decaborano per via rispettivamente endoperitoneale ed inalatoria;

3) le ricerche ematologiche hanno dimostrato, nel ratto e nel cane, significativi aumenti delle emazie, del valore ematocrito, del numero e della percentuale dei polinucleati. L'A. attribuisce alla disidratazione l'aumento a carico delle emazie e del valore ematocrito.

In cani intossicati per via orale con decaborano si è avuto inoltre positività del test di funzionalità epatica alla sulfo-brono-ftaleina, elevati livelli di creatinina urinaria, presenza nelle urine di albumina e di emazie, indici in definitiva di damno epatico e renale, confermato dai reperti autoptici; 
4) Ia restitutio ad integrum negli animali sopravvissuti si attua con notevole ritardo, rispetto a quanto avviene negli intossicati con dose singola. La fenomenologia nervosa e la diminuzione di peso persistono più a lungo rispetto alle alterazioni ematiche;

5) i reperti autoptici possono evidenziare imbrunimento del surrene, congestione del fegato, polmoni, milza; talora fegato grasso ed edema polmonare. Non sono riferiti i reperti istologici.

Miller e Coll, sperimentando su cani e conigli l'azione di un propellente boranico, l'HEF-3, hamno accertato, attraverso dosaggi diretiti, la lunga persistenza dei bolani nel sangue dopo assorbimento acuto, dimostrando come la sostanza sia ancora reperibile a due-tre giorni di distanza dal momento della somministrazione di dosi modeste, e sino a sette giorni dopo la somministrazione di dosi più elevate.

Tali $\Lambda$ A. hamno parimenti confermato come la somministrazione di piccole dosi giornaliere di per se atossiche, se proseguite per sufficiente periodo di tempo, determina, per graduale accumulo, effetti tossici che possono provocare anche la morte. Dopo esposizioni giornalmente protratte per un mese a quantitativi di borani insufficienti a causare segni di tossicità $(100-250 \% / k g$ in cani e conigli) la sostanza può essere repertata in maggiori quantitativi nel fegato ed in quantitativi alquanto inferiori neI S.N.C., in concomitanza con dosaggi serici negativi: l'assenza quindi nel siero di boro in quantità dosabili non esclude l'esistenza dell'accumulo di borani negli organi.

Dumante la fase di ripresa successiva all'intossicazione acuta, piccole ulteriori somministrazioni di per sè prive di conseguenze riproducono il quadro clinico dell'intossicazione in tutta la sua gravità: se però trascorrono sette giorni dall'ultima somministrazione, non è possibile mettere in evidenza tale fenomeno di una maggiore sensibilità residua.

\section{Concentrazioni al limite di tossicità.}

La nozione dell'azione cumulativa dei borani nell'organismo e della conseguente possibilita dell'insorgenza di intossicazione croniche giustifica l'assoluta necessità di prefissare le concentrazioni massime tollerabili dall'uomo nella sua vita lavorativa.

Per il dibolano si è stabilito un numero di M.A.C. di $0,1 \mathrm{mg} / \mathrm{mc}$ (0,1 ppm).

Per il pentaborano si è proposto un valore (provvisorio) di $0,03 \mathrm{mg} / \mathrm{me}$ $(0,01 \mathrm{ppm})$. 
Per il decaborano è stato proposto un valore, parimenti provvisorio, di $0,3 \mathrm{mg} / \mathrm{mc}$, o di $0,05 \mathrm{ppm}$ (American Conference of Governmental Industrial Hygienists, 1957).

I valori proposti per tali M.A.C. tendono a decrescere man mano che si allargano le conoscenze tossicologiche: al 220 Congresso degli Igienisti Industriali Americani (Rochester, 23-26 aprile 1960) per il pentaborano è stato proposto un M.A.C. di 0,005 ppm.

INTOSSICAZIONE DA BORANI NELL'TOMO.

La sintomatologia dell'intossicazione da borani nell'uomo può essere riconosciuta sulla scorta dell'osservazione di Rosendaal (1951), relativa a 4 casi, e soprattutto di Lowe e Freeman (1957), relativa a 83 casi. Inoltre Yiiller e Coll. (1960) hanno riferito i dati relativi ai dosaggi serici del boro in aleuni soggetti esposti. Schoettlin e Coll. (1961) hanno descritto i reperti elettro-encefalografici in 119 soggetti.

Sinora non abbiamo ritrovato nella letteratura alcun caso mortale.

Sintomi dell'intossicazione da diborano sono cefalea, senso di costrizione e bruciore al torace, tosse secea, dolori precordiali, respiro superficiale. L'esame radiografico evidenzia talora infiltrazioni polmonari. Dopo esposizioni più prolungate appaiono anche sintomi a carico del S.N.C. (cefalea, sensazione di ruoto alla testa, vertigini, brivido e, meno frequentemente, febbre); coesiste astenia e debolezza muscolare, persistenti per molte ore. Poco frequenti, sempre localizzati e di breve durata sono i tremori e le contrazioni muscolari fascicolari. Tali sintomi sono simili a quelli che si osservano nell'intossicazione da penta e decaborano, ma di minore gravità e di breve durata, in presumibile rapporto con la notevole velocità con la quale il diborano viene idrolizzato nell'organismo. Quali dati di laboratorio è frequente il reperto di un aumento dell'azoto non proteico e la positività del test di flocculazione alla cefalina.

Sintomi iniziali dell'intossicazione da pentaborano sono rappresentati da cefalea, vertigine, singhiozzo, talora stato stuporoso e nausea post-prandiale, mentre rari sono i sintomi toracici. Incoordinazione, dolori o crampi alle braccia, al collo, al dorso, ai muscoli della coscia sono frequenti; eccezionali gli spasmi di notevole entità dei muscoli addominali. Successivamente si ha la comparsa di contrazioni muscolari intermittenti agli arti ed al collo, tremori, normalmente associati con convulsioni. 
Raramente la sopradescritta sintomatologia si produce rapidamente: molti sintomi a carico del S.X.C. si producono per lo più dopo un apprezzabile periodo di latenza: l'incoordinazione, i tremori e le convulsioni sino a $40-48$ h dopo l'esposizione.

Analoga sintomatologia si ha nell'intossicazione da decaborano, con predominanti efletti sul S.N.C. e con quadri clinici (Lowe e Freeman) in genere di minore gravità.

In complesso i dati di laboratorio nella intossicazione da borani sono espressioni di danno epato-renale e di una probabile distruzione di tessuti, cosa del resto prevedibile sulla scorta degli elementi forniti dalla tossicologia sperimentale. I dati tuttom disponibili sulla funzionalità di tali organi sono suscettibili di ulteriori allargamenti e sviluppi.

L'aumento dell'azotemia e la positività del test di flocculazione alla cefalina sono di frequentissimo reperto non solo nei soggetti con chiari sintomi di intossicazione, ma anche in quelli semplicemente esposti a piccolissime dosi di borani e che non presentano manifestazioni cliniche evidenti: tali indagini riescono quindi di particolare utilità per il depistage dell'intossicazione cronica.

La positività del test alla cefalina è molto precoce (entro 24 h dall'esposizione), ed il ritorno alla norma si ha di regola in 2-4 settimane: meno frequentemente $\mathrm{i}$ valori patologici possono persistere per molti mesi. Analogo è il comportamento dell'azotemia.

La diminuzione dell'escrezione della fenolsulfonftaleina è eccezionale. I valori della bilirubinemia sono di regola normali; le globuline sono frequentemente aumentate. La creatinemia e l'uricemia sono di regola elevate, il che conferma l'ipotesi di una distruzione tossica dei tessuti.

I reperti urinari sono di regola normali tranne che in casi eccezionali (ematuria, albuminuria, glicosuria).

L'esame emocromocitometrico, il valore ematocrito, la sedimetria, il tempo di protrombina forniscono di regola valori normali, non considerando eventuali modifiche legate alla disidratazione o ad infezioni soviapposte. In un solo caso Jowe e Freeman descrivono una persistente lencocitosi, direttamente correlata all'intossicazione da pentabol'ano.

I dosaggi del boro serico (Miller e Coll.) hanno rivelato valori di 0,5-0,6 $\gamma / \mathrm{ml}$ a solo + h di distanza da contaminazioni cutanee accidentali con HEF-3 in quantitativi dell'ordine del $\mathrm{cm}^{3}$, seguite da suecessivo lavaggio con acqua entro pochi minuti. Nei due giorni successivi il boro diminul ed al terzo giorno non fu più reperito. L'intossicazione ebbe decorso asintomatico. 
In 7 lavoratori acutamente intossicati con pentaborano si registrarono nel siero quantitativi di boro compresi tra 0,7 e $1,3 \gamma / \mathrm{ml}$. Campioni raccolti da 18 soggetti che avevano subito esposizioni croniche formirono valori negativi in 15 casi, positivi in 3 casi, con oscillazioni comprese tra 0,15 e $0,22 \gamma / \mathrm{ml}$.

Quanto ai reperti elettroencefalografici negli esposti ad intossicazione accidentale da borani, rinviamo all'esauriente ricerca di Schoettlin e Coll. (1961) che peraltro su 119 soggetti solo raramente hanno trovato variazioni clinicamente significative.

\section{Profilassi.}

Deve essere imposta con estremo rigore data la notevole tossicità dei borani: essa ovviamente consiste in tutto il complesso di norme cautelative per il maneggio e la conservazione dei prodotti; nell'impiego di adeguati indumenti protettivi (maschere, guanti, tute) indispensabili soprattutto nei soggetti esposti a rischio di intossicazione acuta; nella buona ventilazione dei locali, nei quali non dovranno essere raggiunte per lunghi periodi le concentrazioni al limite di tossicità nella selezione e nella assidua vigilanza del personale esposto.

I tests più utili al depistage della intossicazione sono stati prima riferiti.

Si richiama in particolare l'attenzione sulla rapidità con cui si attua l'assorbimento dei borani, quale risulta dalle ricerche di Miller e Coll. Ne deriva l'assoluta necessità di immediati e prolungati lavaggi con acqua corrente della cute contaminata e dell'immediato ricambio degli indumenti personali eventualnente lordati.

Da tenersi presente che la minima concentrazione di tali sostanze nell'aria apprezzabile dall'olfatto prima riferita $(2-4 \mathrm{mg} / \mathrm{mc}$ per il diborano; 2,5 mg/me per il pentaborano; $0,3 \mathrm{mg} / \mathrm{mc}$ per il decaborano) è troppo elevata per poter fornire allorganismo una utile spia del rischio cui è esposto: solo il decaborano può essere apprezzato per via olfattoria nell'aria a livelli sub-tossici.

Non esiste una terapia specifica dell'intossicazione da borani: il trattamento degli intossicati sarà pertanto sintomatico.

Il dimercaptolo (BAL) ed i sostituti dell'atropina sono praticamente inefficaci. Gruppi di animali esposti al diborano e quindi trattati con atropina, benadryl, altri anti istaminici e con nembutal hanno dimostrato, rispetto ai controlli non trattati, una lieve, ma non significativa diminuzione del percento di mortalità (Krackow). 
DERIVATI METILATI DELL'IDRAZLA DIMETIL-IDRAZINA ASMMMETRICA.

Tra i derivati metilati dell'idrazina, la dimetil-idrazina asimmetrica è quella che trova massimo impiego come propellente ad alto potere eneregetico.

I derivati dell'idrazina sono conosciuti sin dal 1875, mentre l'idrazina pura è nota solo dal 1895. Tale ritardo nell'isolare il materiale puro è dovuto alla sua grande reattività ed instabilità.

L'idrazina ed i suoi derivati metilati (metil-drazina, dimetil-idrazina simmetrica, asimmetrica) sono composti dotati di elevatissima tossicità, che attualmente è nota sopratutto dai dati di tossicologia sperimentale rilevati sugli animali. I dati disponibili nella letteratura (Jacobson e Coll., 1955) parlano per una tossicità comparativa dell'idrazina e dei suoi derivati sostanzialmente (salvo piccole differenze) qualitativamente uguale, ma quantitativamente diversa, con progressivo ammento dall'idrazina alla dimetil-idrazina simmetrica, alla asimmetrica ed alla metilidrazina, che rappresenta il derivato fornito di maggiore tossicità.

Caratteristiche FIsiche e chmiche.

La dimetil-idrazina asimmetrica si presenta come un liquido incolore, di odore ammoniacale, igroscopico, miscibile con tutti i solventi compresa lacqua. Ha peso molecolare $=60$, punto di congelamento pari a - 520 C; punto di ebollizione pari a $+63^{\circ} \mathrm{C}$; densità a $22^{\circ} \mathrm{C}=0,7914$.

TOSSICOLOGIA SPERIMENTALE.

La tossicologia dei derivati idrazinici è nota sopratutto dai dati sperimentali in quanto mancano ancora osservazioni sull'uomo, se si eccettuano quelle relative a lesioni locali (dermatiti da contatto, causticazioni) determinate sopratut to dall'idrazina.

Vie di penetrazione.

La dimetil-idrazina asimmetrica produce negli animali intossicazioni per qualsiasi via venga introdotta (percutanea, inalatoria, orale, ipodermica). La via di introduzione non risulta avere una notevole 
influenza sul grado di tossicità (Witkin, 1956) il che denota un completo assorbimento attraverso l'apparato digerente ed una bassa velocità di detossificazione e di eliminazione. Ne deriva che piccole dosi ripetute hanno azione cumulativa e possono condurre all'intossicazione cronica.

Applicata sulla cute è molto meno tossica localmente della idmaina, che ha effetto caustico molto più accentuato, presumibilmente dornto alla sua basicità. Comunque applicazioni ripetute possono condurre a dermatiti. Pur senza notevoli effetti locali le applicazioni percutanee determinano l'assorbimento della sostanza e possono causare intossicazioni sistemiche (Rothberg, Cope).

\section{Tossicità acuta.}

La $\mathrm{LD}_{50}$ per la dimetil-idrazina asimmetrica è pari a $60 \mathrm{mg} / \mathrm{kg}$ per via endovenosa nel cane; a $250-290 \mathrm{mg} / \mathrm{kgr}$ nel topo, con piccole variazioni in rapporto alla via di introduzione (orale, endovenosa, endoperitoneale); da 119 a $131 \mathrm{mg} / \mathrm{kg}$ nel ratto (Witkin, 1956), con analoghe piccole variazioni. Per i tre animali considerati la dimetil-idrazina rappresenta il prodotto acutamente più tossico; per il cane ed il ratto segue nell'ordine l'idrazina, la dimetil-idrazina asimmetrica e la forma simmetrica; per il topo la dimetil-idrazina simmetrica, l'idrazina e la dimetil-

Tabella $3-$ LD $_{50}$ PER VIa INALATORIa per esposizioni di 4 ORE A VAPORI di derivati idrazixici (Da Jacobson e Coli., 1955).

\begin{tabular}{|c|c|c|c|}
\hline \multirow{2}{*}{ Derivato idrazinico } & \multicolumn{3}{|c|}{$\mathrm{LD}_{50}$} \\
\hline & & ppm & $\mathrm{mg} / \mathrm{m}^{3}$ \\
\hline $\begin{array}{l}\text { Idrazina } \\
\text { Ratto } \\
\text { Topo }\end{array}$ & $\begin{array}{l}570 \\
252\end{array}$ & da $504+649$ & $\begin{array}{l}750 \\
330\end{array}$ \\
\hline $\begin{array}{l}\text { Metil-idrazina } \\
\text { Ratto } \\
\text { Topo } \\
\text { Criceto }\end{array}$ & $\begin{array}{r}74 \\
56 \\
143\end{array}$ & $\begin{array}{llll}\text { da } & 71 & \text { a } & 78 \\
\text { da } & 50 & \text { a } & 110 \\
- & - & - & -\end{array}$ & $\begin{array}{l}139 \\
105 \\
270\end{array}$ \\
\hline $\begin{array}{c}\text { Dimetil-idrazina as. } \\
\text { Ratto } \\
\text { Topo } \\
\text { Criceto }\end{array}$ & $\begin{array}{l}2 j 2 \\
172 \\
392\end{array}$ & $\begin{array}{llll}\text { da } & 219 & \text { a } & 290 \\
\text { da } & 150 & \text { a } & 194 \\
\text { da } & 376 & \text { a } & 413\end{array}$ & $\begin{array}{l}618 \\
423 \\
962\end{array}$ \\
\hline $\begin{array}{c}\text { Dimetil-idrazina sim. } \\
\text { Ratto }\end{array}$ & - & da 280 a 400 & 一 \\
\hline
\end{tabular}


idrazina asimmetrica. La $\mathrm{LI}_{50}$ per via inalatoria dopo esposizioni di 4 ore a vapori di derivati idrazinici è riferita nella Tabella 3.

Segli animali esposti acutamente all'azione dei derivati idrazinici si ha anoressia, salivazione, vomito, esoftalmo, dispnea e convulsioni: i segni di eccitazione nervosa e le convulsioni compaiono più facilmente negli animali esposti alla metil-idrazina che rispetto agli altri derivati presenterebbe (Jacobson e Coll., 1955) una azione emolitica più spicarata (anemia, comparsa di emoglobina plasmatica, reticolocitosi, iperbilirubinemia).

All'esame anatomo-patologico si notano segni di edema polmonare con presenza talora di fatti emorragici. Petecchie emorragiche sono state anche rilevate a carico dell'encefalo e del midollo spinale. Nell'intossicazione acuta mancano le alterazioni epatiche che sono invece presenti nell'intossicazione cronica.

In complesso a carico del sistema nervoso centrale si nota ipereccitabilità seguita da convulsioni tonico-cloniche accompagnate talvolta da opistotono: durante le convulsioni, che sono controllabili con i comuni anticonvulsivanti le pupille sono midriatiche.

A carico dell'apparato respiratorio si ha dispnea, cianosi ed altị segni di edema polmonare.

A carico dell'apparato circolatorio può notarsi ipotensione, rallentamento della conduzione atrio-ventricolare, diminuzione della capacità contrattile del cuore e fibrillazione ventricolare.

L'apparato digerente dimostra segni di ipertonia, presumibilmente attribuibili al S.N.C.

In roncordanza con i reperti anatomo-patologici mancano nelle forme acute disturbi riferibili ad insufficienza epatica, calatteristici delle forme croniche.

\section{Tossiciti subacuta e cronica.}

Discretamente nota per l'idlazina su cui vertono la maggior parte delle ricerche esistenti, è assai meno nota per i suoi derivati metilati, e, in particolare, per la dimetil-idrazina asimmetrica.

Felle forme croniche si ha comunque un netto predominio dei segni di compromissione del fegato che presenta estesi fenomeni di degenerazione grassa interessanti principalmente le porzioni centrali di ciascun lobulo e che colpiscono scarsamente i vasi sanguigni e $\mathrm{i}$ dotti biliari. Le cellule epatiche sembrano perdere il loro citoplasma, residuandone nuclei praticamente denudati. Nei casi più lievi le lesioni possono essere reversibili. 
Tali alterazioni sono ravvicinabili a quelle che si osservano nell'intossicazione da fosforo, nella quale però esse sono uniformemente diffuse a tutti gli organi e non localizzate prevalentemente al fegato.

Coesistono fatti degenerativi a carico del rene con conseguente albuminuria.

Secondo Underlill nell'avvelenamento idrazinico si ha ipoglicemia dovuta ad un aumento dell'ossidazione del glucosio. is pure descritto un aumento degli aminoacidi nel plasma.

\section{Concentrazioni al limite di tossicità.}

La massima concentrazione tollerabile nell'aria per esposizioni croniche per la idrazina e per i suoi derivati metilati non è stata ancora prefissata.

Sulla scorta degli esperimenti di tossicità acuta, subacuta e cronical sugli animali Comstock e Coll., 1953, avevano proposto un M.A.C. di 6 $\mathrm{mg} / \mathrm{m}^{3}$ pari a 5 ppm per l'idrazina.

Al $22^{\circ}$ Congresso degli Igienisti Industriali americani (Rochester, 23-26 aprile 1960) è stato proposto un M. A.C. di $1,3 \mathrm{mg} / \mathrm{m}^{3}$ pari a $1 \mathrm{ppm}$. Autori russi propongono un M.A.C. di $0,1 \mathrm{mg} / \mathrm{m}^{3}$.

Evidentemente per la dimetil-idrazina asimmetrica, che è dotata di maggior tossicità, è da prevedere un M.A.C. ancora inferiore.

Le concentrazioni minime di tali sostanze svelabili nell'aria per le loro caratteristiche olfattorie (odore ammoniacale) è (Jacobson e Coll., 195̃5) di 3-4 ppm per l'idmazina; di $1-3 \mathrm{ppm}$ per la metil-idmazina; di 6-14 ppm per la dimetil-idrazina asimmetrica.

Poichè tali concentrazioni (salvo che per esposizioni croniche protratte ai derivati idrazinici) sono praticamente vicine ai limiti di tossicità, l'odorato rappresenta una preziosa spia onde evitare l'esposizione e concentrazioni acutamente o subacutamente tossiche di tali sostanze.

IN'TOSSICAZIONI NELL'LOMO.

Poichè, come si è detto, mancano a tutt'ora estese osservazioni nell'uomo, la sintomatologia dell'intossicazione acuta, dedotta dai dati sperimentali negli animali, dovrebbe comunque consistere in dermatiti, congiuntiviti, cefalee, anoressia, perdita di peso, nausea, vomito, anemia, convulsioni.

Prevalenza di segni di damno epato-renale nelle forme croniche. 


\section{Profitassi.}

Gli addetti al maneggio ed alle lavorazioni con dimetil-idrazina asimmetrica dovramno indossare indumenti protettivi (maschere, occhiali, granti, grembiule di gomma). Eventuali schizzi vanno lavati abbondantemente con acqua. Hassima cura si dovà avere per la ventilazione dei locali.

Il trattamento degrli intossicati è puramente sintomatico, non conoscendosi antidoti. Contro le convulsioni, utili gli anticonrulsivanti (barbiturici, dintoinici). Le ustioni vanno trattate come se fossero prodotte dal calore o da alcali. I danni epatici e renali possono essere valutati con indagini cliniche e funzionali diverse e trattati con somministrazione di aminoacidi, acido ascorbico, piruvato, e, con cautela, con glucosio.

\section{BIBLIOGRAFIA}

Cole V. V. - IIll J. L. - Orkexus A. II., Problems in the Study of Decaborane and Possible Therapy of Its Poisoning. "A.M.A. Arch. Ind. Hyg. and Occupational Merl. ", 10, 158, (1954).

Comstock C. C. - Lawson I. II. - Green E. A. - OBerst F. W., Inhalation Toxicity of IIydrazine vapor. "A.M.A. Arch. Ind. Hyg. ", 10, 476, (1954).

Constock C. C. - OвERsт F. W., The median detectable concentration of diborane, pentaborane and decaborane by odor for man. U.S. Chemical Corps Med. Lab. Research Report 11. 206, Agosto 1953.

Dible W. - Truog E. - Berger K., Boron Determination in Solis and Plants. "Anal. Chem. ", 26, 418-421, (1954).

Ellis G. - Zook E. - BAudicn O., Colorimetrie Determination of Boron Using 1, L'Dianthrimide. "Anal. Chem. ", 21, 1345-1348, (1949).

Intcier J. - Wrucose L., Colorimetrie Determination of Boron Using Carwine. "Anal. Chem. ", 22, 567-569, (1950).

IItll L. D.G - IBsox E. T. - FAK H. C., The Brealdown of Decaborane in Irotein Solutions, Vater and Alcohol. M.L.R.R. n. 324, Army Chem. Center, Mil., 1954.

Iill W. H. - Svirbely J. L., Gas Mask Protection Against Decaborane. "A.ML.A. Arch. Ind. Hyg. ", 10, 69, (1954).

HILL W. II. et Al., Analysis of Biological IIaterials for Boran. "Arch. Ind. Health ", 17, 210, (1958). 
Hurd D. T., An Introduction to the Chemistry of the Hydrides. New Jork, Joln Wiley \& Sons, Inc. 1952.

Jacoboss K. H. - Clem J. II. - WiteeludrigitT Jr. - RiNeitart W. E. Mares N., The Acute Toxicity of the Tapores of Some Methylated Hydrazine Deriratives. "A.M.A. Arch Ind. Health. », 12. 609, (1955).

Kuxkel A. M., Literature Survey on Toxicity of Boric Acid and Sodium. Tetraborate (Borace). M.D.S.K. n. 2, Army Chem. Center Md., 1950.

Krackow E. H., Toxicity and ITealth IIazards of Boron IIydrides. "A.M.A. Areh. Ind. Hygr. ", 8, 335, (1953).

Krop S., Toxicology of IIydrazine: A. Review. "A.M.A. Areh. Ind. Hyg. ", 9. $199,(1954)$.

Levinskas G. - Poidis A. - Novick W., Distribution and Excretion of Boranes. "A.M.A. Arch. Ind. Health », 14, 346-349, (I956).

Lowe II. J. - Freenax G., Boron Iydride (Boraco) Inlorication in Man. "A.M.A. Arch. Ind. Health ", 16, 523, (1957).

Miller D. G. - Tamas A. - Robinson I. A Simple Clinical Test for Boron Mydride Exposure WADC Technical Report 59-123, Wrimht Patterson Air Force Base, Ohio, April 1959.

Miller F. - Tamas A. - Robinson I. - Merriweatier E., Cumulative Effects of Borane Toxicity. As Revealed by a Clinical Test. Wadd Technical Report 60-604, Wright Air Development Division, August 1960.

Naftel J., Colorimetric Nicro determination of Boron. "Ind. Eng. Chem. Anal. Ed. ", 11, 407-409, (1939).

Powell W. - Hordcastle E. - Poindexter H., Curcumin Method for Spectrophotometric Determination of Boron. CCC 1024, TK 230, March 1957.

Rincitart W. - Donati E. - Greene E. A., The sub-acute and chronic Toxicit of 1,1-dimethylydrazine vapor. "Am. Ind. Hyg. Ass. J. ", 21, 207, (1960).

Rosendant II. M., Clinical Observations on the Toxicology of Boron Iydrides. "A.M.A. Arch. Ind. Hyg. and Occupational Med. ", 4, 257, (1951).

Schoetthis C. E. - Cinnino G. M. - Walter R. D. - Freedmax T., Toxicological Research On Central Nervous System Elfects of Borane Fuels. "ASD. Tech. Report ", 61, 438, (1961).

Spicer G. - Stricklavd J., Compounds of Curcumin d Boric A cid. "J. Chem. Soc. ", 4644, 4650, 4656, (1952).

Spicer G. - Stricklaxd J., The Determination of Microgram and Sub Jicrogram Amounts of Boron. "ANA. Chin. ACTA ", 18, 231-523, (1958).

STock A. E., Hydrides of Boron and Silicon. Ithaca, N. J. Cornell I'niversity Press., (1.933).

Svirbely J. L., Acute Toxicity Studies of Decaborne and Pentaborane by Inhalation. "A.M.A. Arch. Ind. IIyg. ", 10. 298, (1954). 
Svikbey J. L., Subacute Toxicity of Decaborane and Pentaborane Vapors. "A.M.A. Arch. Ind. Hyg. ", 10, 305, (1954).

Svinuely J. L., Toxicity Test of Decaborane for Laboratory Animals. I. Acute Toxicity Studies. "A.M.A. Arch. Ind. Healt ”, 11, 132, (1955).

Svinber J. L., Toxicity T'est of Decaborane for Laboratory Animals. II. ElJect of Repeated Dosee. "A.M.A. Arch. Ind. Healt ", 11, 138, (1955).

Thuexes C. II. - Roth H. P. - Swensar E. A. - Morgan C. V. - Lombard C. F., Progress Report on Acute and Chronic Toxicities of IIydrazine. Nortl American Aviation, Ine. Dept. AI 731, June 1948.

WiLLs J. II., Toricity and Pharmacology of Boron Hydrides. 7MDSK n. 15, Army Chemical Center, Md. 1953. 